\title{
Blood product transfusion in liver transplantation in First Clinical Hospital of Mongolia
}

\author{
Bazarragchaa Regjii', Tseyenpiljee Amgaa', Bayalagmaa Khuvtsagaan ${ }^{1}$, Chuluunbaatar Donkhim¹, Ganbold Lundeg ${ }^{2}$ \\ ${ }^{1}$ Department of Anesthesia, First Central Hospital of Mongolia, Ulaanbaatar, Mongolia \\ ${ }^{2}$ Department of Emergency Care and Anesthesia, Mongolian National University of Medical Science, Ulaanbaatar, Mongolia
}

Background: Liver transplantation is a well-accepted treatment of end-stage liver diseases. Seven-eight liver transplants have been performed from 2011 to June of 2019 in First Clinical Hospital of Mongolia. Numerous advances in perioperative management, like expertise in surgical techniques, better preoperative optimization, intraoperative monitoring and management, changes in immunosuppression regime and advances in postoperative management, not only increased the number of this procedure but also the outcome. The role of the anesthesiologist is to provide safe anesthesia and maintain an acceptable hemodynamic performance, ensuring sufficient perfusion to the vital organs. Estimation of the amount of blood products required during liver transplantation can help provision of adequate blood supply, minimize transfusion associated complications and plan for preventive measures in high risk patients.

Methods: Our objective is to investigate some factors impact to perioperative blood product transfusion. We used data of patients who underwent liver transplantation between October 2011 and June 2019 at First Clinical Hospital of Mongolia, were reviewed. The all amount of blood product utilized during surgery and some factors, including pretransplant laboratory data, pretransplant clinical data were recorded.

Results: We studied 77 patients who underwent liver transplantation. The mean \pm standard deviation amounts of red blood cells and fresh frozen plasma transfusion during surgery were $1.64 \pm 2.36$ and $2.22 \pm 2.7$ liters, respectively. The mean amount of red blood cells and albumin was significantly $(P<0.003$ and $P<0.005)$ correlated with model for end-stage liver disease $(M E L D)$ score of patients. The mean amount of blood products utilized during operation was decreased from 2015 to 2019 except two retransplantation patients.

Conclusions: Some preoperative factors may predict blood transfusion requirements in patients undergoing liver transplantation. Therefore, evaluation of patients before operation should be considered to provide adequate blood supply. Understanding preoperative factors associated with rate of transfusion may help us to best utilize the limited available blood resources. 